Journal Wetenskap Health

\title{
The Function of Immunology, Antigen and Antibody
}

\author{
Tarek Mohsen \\ Faculty of Medicine, Ain Shams University, Egypt
}

\begin{abstract}
The aims of the study is the function of , imonology, antigen and antibody. The discussion is the function of antigen and also the types of antigen The type of antigen is based on its determinant: (a) Unideterminant, (b) Unideterminant, (c) Multideterminant (d) Multideterminant. The type of antigen based on the specifics : (a) Heteroantigen (b) Xenoantigen (c) Alloantigen (d) Specific organ antigens (e) Autoantigen . Types of antigens based on their chemical content: (a) Carbohydrates, (b) Lipids (c) Nucleic (d) Protein . (Antibodies have the ability to recognize and attach / attach to antigens that are thought to cause disease by the body and recognizing and attaching themselves to antigens, the antibody substance always acts as a marker, and then sends a signal to other white blood cells to attack the foreign substance
\end{abstract}

Keywords: Immunology, Antigen, Antibody

\section{Introduction}

Immunology includes prevention. Prevention itself is deliberately providing immunity or immunity, so that the child, even though he later gets an infection, will not die or suffer from disabilities (sequilae). In general, antigens can be classified into two main types, namely exogenous antigens and endogenous antigens. Exogenous antigens are antigens that are presented from outside to the host in the form of microorganisms, pollen, drugs or pollutants. This antigen is responsible for a spectrum of human diseases, from infectious diseases to diseases that are treated immologically, such as asthma. (Bezirtzoglou \& Stavropoulou, 2011).

The specificity of the reaction between antigen and antibody has been demonstrated through studies conducted by Landsteiner. It combines organic radicals to proteins and produces antibodies against these antigens. The decision obtained shows that antibodies can distinguish between different groups of proteins or the same chemical group but different positions.

\section{Definition of Immunology}

Immunology is a broad branch of biomedical science that includes the study of all aspects of the immune system (immunity) in all organisms (Pradeu, 2011). Immunology, among others, studies the physiological role of the immune system in both health and illness; immune system malfunction in immunological disorders (autoimmune disease, hypersensitivity, immune deficiency, allograft rejection); physical, chemical, and physiological characteristics of the immune system components in vitro, in situ, and in vivo. Immunology has various applications in various disciplines and is therefore broken down into sub-disciplines (Fagan, 2007).

Immunology was originally a branch of microbiology that studied the body's response, especially the immune response, to infectious diseases. contagion theory which states that in infectious diseases there is a substance that can transfer the disease from one individual to 
another, but the substance is so small that it cannot be seen with the eye and at that time cannot be identified.

\section{History of Immunology Development}

In 1798, Edward Jenner observed that a person could naturally avoid variola infection if he had been previously exposed to cow pox (cow pox). Since then, smallpox vaccine was used, although at that time it was not known how the actual mechanism occurred. Pasteur in 1880 discovered the causes of infectious diseases and was able to breed microorganisms and established the germ theory of disease. This discovery was then followed by the human rabies vaccine in 1885 .

Pasteur's work then became the basis for further vaccine development, which was a brilliant achievement in the field of immunology which had a positive impact on reducing morbidity and mortality of infectious diseases in children. In 1880, Robert Koch discovered the germs that cause tuberculosis. In order to find a vaccine against tuberculosis, he observed a tuberculin reaction (1891) which is a slow hypersensitivity reaction of the skin against tuberculosis germs. This tuberculin reaction was then used by Mantoux (1908) to diagnose tuberculosis in children. Immunology began to be used to diagnose the disease in children. The vaccine against tuberculosis was discovered in 1921 by Calmette and Guerin, known as the BCG vaccine (Bacillus Calmette-Guerin). It was later discovered that not only live microorganisms can induce immunity, even nonliving materials can induce immunity. After Roux and Yersin discovered diphtheria toxin in 1885, Von Behring and Kitasato discovered diphtheria antitoxin in animals (1890). Since then, treatment with immune serum obtained from horses has been initiated and immunology is applied in the treatment of infectious diseases in children.

Treatment with immune serum later evolved into treatment with specific immunoglobulins or gamma globulin obtained from humans. With the use of immune serum, clinical abnormalities appear due to administration of this serum. Two pediatricians, Clemens von pirquet from Austria and Bela Shick from Hungary reported in 1905, that children who received injection of immune serum from horses sometimes suffer from fever.

In 1911-1914, Noon and Freeman tried to treat hay fever by means of immune therapy, which involves injecting small amounts of subcutaneous pollen. Basically at that time it was thought that pollen excretes toxins, with the hope that neutralizing antitoxins will be formed. Since then this method is still used to treat allergic diseases to certain antigens, known as desensitization. However, the mechanism currently adopted is based on the formation of blocking antibodies.

With the discovery of the tuberculin reaction, Schloss (1912) and von Pirquet (1915) performed a scratch test on the skin for the diagnosis of allergic disease in children. Talbot (1914), a pediatrician, with a scratch test saw a relationship between childhood asthma and eggs. Cooke (1915) modified the scratch test with the intracutaneous test, and also reported that heredity plays a role in allergic disease. In 1913, Shick also introduced a skin test to determine a person's susceptibility to diphtheria, so that more and more immune phenomena were applied in diagnostic tests for childhood diseases.

Rackemann (1918) saw that most asthma in children has an allergic basis and is called extrinsic type asthma. Prausnitz and Kustner (1921) stated that substances that cause skin sensitization in skin tests can be transferred through patient serum. At that time, the exact mechanism of allergy was not known. Now, thanks to the research that has been done, the cellular and molecular processes that occur in allergic diseases can be described. With the 
discovery of complement by Bordet (1894), diagnostic tests using immune phenomena developed again with the complement fixation test (1901), as in syphilis. clinical disorders based on immune reactions became increasingly recognized. At that time, the immune phenomenon that occurred could only be described in terms of immunology. It was not until 1939, 141 years after Jenner's discovery, that Tiselius and Kabat discovered electrophoretically that antibodies were located in the gamma globulin spectrum which was later called immunoglobulin ( $\mathrm{Ig})$. By means of immunoelectrophoresis, it is known that immunoglobulins consist of 5 classes, namely $\operatorname{IgA}, \operatorname{IgG}, \operatorname{IgM}, \operatorname{IgD}$ and $\operatorname{IgE}$ (WHO, 1964), and then it is known that each of these classes has sub-classes. In 1883, Metchnikoff actually said that the body's defenses were not only played by humoral factors, but leukocytes also played a role in the body's defense against infectious diseases. At that time, the role of leucocytes was only known for its phagocytosis function. It was he who discovered the macrophage cells. We now know that macrophage cells actively play a role in cellular immunity for antigen elimination.

\section{Defenition of Antigen}

Antigen is a known foreign material and is a target that will be destroyed by the immune system. (Glennie \& van, 2003). Antigens are found on the surface of all cells, but under normal circumstances, a person's immune system does not react to his own cells. So it can be said that the antigen is a substance that stimulates an immune response, especially in the production of antibodies. (Oriol et al. 1993). Antigens are usually proteins or polysaccharides, but can also be any other molecule, including the small molecule (hapten) attached to the carrier-protein. The immune system or immune system is a system of protection from biological outside influences carried out by specific cells and organs in an organism. If the immune system is working properly, it will protect the body against bacterial and viral infections, as well as destroy cancer cells and other foreign substances in the body.

\section{Types of Antigents}

According to Raghunathan (2012). The type of antigen is based on its determinant: (a) Unideterminant, univalent, is a type of epitope one and the number is one, (b) Unideterminant, multivalent, is a type of epitope one, the number is more than one, (c) Multideterminant, univalent, is a type of epitope more than one and the number is one, (d) Multideterminant, multivalent, is a type of epitope more than one, the number of more than one (Stephenson et al. 1974).

The type of antigen based on the specifics : (a) Heteroantigen $\rightarrow$ possessed by many species, (b) Xenoantigen $\rightarrow$ belongs to certain species, (c) Alloantigen $\rightarrow$ belongs to one species (d) Specific organ antigens $\rightarrow$ possessed by certain organs, (e) Autoantigen $\rightarrow$ comes from his own body. (Revelen et al. 2000). Types of antigens based on dependence on T cells: (a) $\mathrm{T}$ dependent is about the antigen that needs recognition of $\mathrm{T}$ cells and $\mathrm{B}$ cells to stimulate antibodies, (b) Independent $\mathrm{T}$ is about the antigen that can stimulate $\mathrm{B}$ cells without knowing $\mathrm{T}$ cells first (Abe et al. 2011). Types of antigens based on their chemical content: (a) Carbohydrates are immunogenic, (b) Lipids: not immunogenic is a hapten, (c) Nucleic acids are non-immunogenic antigens, (d) Protein is immunogenic. (Copland et al. 2005)

\section{Antigen Mechanism}

In the environment around us there are many small molecular substances that can enter the body. These small substances can become antigens when they attach to our body's proteins. The small substance that can turn into an antigen is known as a hapten. These substances escape the non-specific response barrier (external or internal), then these 
substances enter and bind to B lymphocytes which will synthesize the formation of antibodies. The antigen that enters the body will bind to B lymphocyte receptors.( Heesters et al. 2016). This binding causes B lymphocytes to differentiate into plasma cells. The plasma cells will then form antibodies that are able to bind to antigens that stimulate the formation of antibodies themselves.

\section{Understanding Antiboody}

Antibody and gamma globulin) are glycoproteins with certain structures secreted from B-lymphocyte absorbers that have been activated to become plasma cells, in response to certain antigens and reactive to these antigens. The human immune system is determined by the body's ability to produce antibodies to fight antigens. Antibodies can be found in the blood or glands of other vertebrates, and are used by the immune system to identify and neutralize foreign bodies such as bacteria and viruses. Antibody molecules circulate in blood vessels and enter body tissues through an inflammatory process. They are made of a few basic structures called chains. Each antibody has two large and two light chains.

There are several different types of antibody heavy chains, and several different types of antibodies, which are assigned to different classes (en: isotype) according to each heavy chain. Five different antibody isotypes are known to exist in mammals and play different roles and help direct the appropriate immune response for each different type of foreign body that enters the body, namely: $\operatorname{IgG}, \operatorname{IgM}, \operatorname{IgA}, \operatorname{IgD}$ and $\operatorname{IgE}$, which have different areas. C. If the immune system is weakened, its ability to protect the body is also reduced, causing pathogens, including viruses that cause colds and flu, to develop in the body. The immune system also provides surveillance of tumor cells, and inhibition of this system has also been reported to increase the risk of developing some cancers. In fact, the power of a person's antibody against antigens contained in a person's body. Antibodies are proteins that can be found in the blood or glands of other vertebrates, and are used by the immune system to identify and neutralize foreign bodies such as bacteria and viruses. They are made of a few basic structures called chains. Each antibody has two large heavy chains and two [light chains]. Antibodies are produced by a type of blood cell called B cells.

\section{The Function of Antibody}

Antibodies have the ability to recognize and attach / attach to antigens that are thought to cause disease by the body. ( $\mathrm{Lu}$ et al. 2018). In recognizing and attaching themselves to antigens, the antibody substance always acts as a marker, and then sends a signal to other white blood cells to attack the foreign substance.

\section{Conclusion}

Immunology is a branch of microbiology that studies the body's response, especially the immune response, to infectious diseases. In 1546, Girolamo Fracastoro proposed a contagion theory which states that in infectious diseases there is a substance that can transfer the disease from one individual to another, but the substance is so small that it cannot be seen with the eye and at that time cannot be identified. The antigen is a known foreign material and is a target that will be destroyed by the immune system. So it can be said that the antigen is a substance that stimulates an immune response, especially in the production of antibodies. Antigens are usually proteins or polysaccharides, but can also be any other molecule, including the small molecule (hapten) attached to the carrier-protein. The immune system or immune system is a system of protection from biological outside influences carried out by specific cells and organs in an organism. Antibodies (English: antibody, gamma globulin) are glycoproteins with certain structures secreted from B-lymphocyte absorbers that have been 
activated to become plasma cells, in response to certain antigens and reactive to these antigens.

\section{References}

Abe, R., Ohashi, H., Iijima, I., Ihara, M., Takagi, H., Hohsaka, T., \& Ueda, H. (2011). "Quenchbodies": quench-based antibody probes that show antigen-dependent fluorescence. Journal of the American Chemical Society, 133(43), 17386-17394.

Bezirtzoglou, E., \& Stavropoulou, E. (2011). Immunology and probiotic impact of the newborn and young children intestinal microflora. Anaerobe, 17(6), 369-374.

Copland, M. J., Rades, T., Davies, N. M., \& Baird, M. A. (2005). Lipid based particulate formulations for the delivery of antigen. Immunology and cell biology, 83(2), 97105.

Fagan, M. B. (2007). The search for the hematopoietic stem cell: social interaction and epistemic success in immunology. Studies in History and Philosophy of Science Part C: Studies in History and Philosophy of Biological and Biomedical Sciences, 38(1), 217-237.

Glennie, M. J., \& van de Winkel, J. G. (2003). Renaissance of cancer therapeutic antibodies. Drug discovery today, 8(11), 503-510.

Heesters, B. A., van der Poel, C. E., Das, A., \& Carroll, M. C. (2016). Antigen presentation to B cells. Trends in immunology, 37(12), 844-854.

Lu, L. L., Suscovich, T. J., Fortune, S. M., \& Alter, G. (2018). Beyond binding: antibody effector functions in infectious diseases. Nature Reviews Immunology, 18(1), 46.

Oriol, R., Ye, Y., Koren, E., \& Cooper, D. K. (1993). Carbohydrate Antigens Of Pig Tissues Reacting With Human Natural Antibodies As Potential Targets For Hyperacute Vascular Rejection In Pig-To-Man Organ Xenotransplantation1. Transplantation, 56(6), 1433-1442.

Pradeu, T. (2011). The limits of the self: immunology and biological identity. Oxford University Press.

Raghunathan, G., Smart, J., Williams, J., \& Almagro, J. C. (2012). Antigen-binding site anatomy and somatic mutations in antibodies that recognize different types of antigens. Journal of Molecular Recognition, 25(3), 103-113.

Revelen, R., Bordron, A., Dueymes, M., Youinou, P., \& Arvieux, J. (2000). False positivity in a cyto-ELISA for anti-endothelial cell antibodies caused by heterophile antibodies to bovine serum proteins. Clinical chemistry, 46(2), 273-278.

Revelen, R., Bordron, A., Dueymes, M., Youinou, P., \& Arvieux, J. (2000). False positivity in a cyto-ELISA for anti-endothelial cell antibodies caused by heterophile antibodies to bovine serum proteins. Clinical chemistry, 46(2), 273-278.

Stephenson, J. R., Tronick, S. R., \& Aaronson, S. A. (1974). Analysis of type specific antigenic determinants of two structural polypeptides of mouse RNA C-type viruses. Virology, 58(1), 1-8. 